\title{
Paracrine Mechanisms of Mesenchymal Stromal Cells in Angiogenesis
}

\author{
Selma Maacha, ${ }^{1}$ Heba Sidahmed ${ }^{D},{ }^{2}$ Shana Jacob, ${ }^{1}$ Giusy Gentilcore, ${ }^{2}$ Rita Calzone, ${ }^{2}$ \\ Jean-Charles Grivel, ${ }^{1}$ and Chiara Cugno ${ }^{2}{ }^{2}$ \\ ${ }^{1}$ Deep Phenotyping Core, Sidra Medicine, Doha 26999, Qatar \\ ${ }^{2}$ Advanced Cell Therapy Core, Sidra Medicine, Doha 26999, Qatar \\ Correspondence should be addressed to Chiara Cugno; ccugno@sidra.org
}

Received 4 October 2019; Accepted 5 February 2020; Published 9 March 2020

Academic Editor: Joery De Kock

Copyright (C) 2020 Selma Maacha et al. This is an open access article distributed under the Creative Commons Attribution License, which permits unrestricted use, distribution, and reproduction in any medium, provided the original work is properly cited. The publication of this article was funded by Qatar National Library.

\begin{abstract}
The role of the mesenchymal stromal cell- (MSC-) derived secretome is becoming increasingly intriguing from a clinical perspective due to its ability to stimulate endogenous tissue repair processes as well as its effective regulation of the immune system, mimicking the therapeutic effects produced by the MSCs. The secretome is a composite product secreted by MSC in vitro (in conditioned medium) and in vivo (in the extracellular milieu), consisting of a protein soluble fraction (mostly growth factors and cytokines) and a vesicular component, extracellular vesicles (EVs), which transfer proteins, lipids, and genetic material. MSC-derived secretome differs based on the tissue from which the MSCs are isolated and under specific conditions (e.g., preconditioning or priming) suggesting that clinical applications should be tailored by choosing the tissue of origin and a priming regimen to specifically correct a given pathology. MSC-derived secretome mediates beneficial angiogenic effects in a variety of tissue injuryrelated diseases. This supports the current effort to develop cell-free therapeutic products that bring both clinical benefits (reduced immunogenicity, persistence in vivo, and no genotoxicity associated with long-term cell cultures) and manufacturing advantages (reduced costs, availability of large quantities of off-the-shelf products, and lower regulatory burden). In the present review, we aim to give a comprehensive picture of the numerous components of the secretome produced by MSCs derived from the most common tissue sources for clinical use (e.g., AT, BM, and CB). We focus on the factors involved in the complex regulation of angiogenic processes.
\end{abstract}

\section{Introduction}

Mesenchymal stromal cells (MSCs) were described for the first time in 1970 by Alexander Friedenstein as a "population of bone marrow stromal cells capable of mesodermal differentiation and trophic support of hematopoiesis" $[1,2]$. Mesenchyme is derived from Greek, meaning "middle" (meso) "infusion," and it refers to the ability of mesenchymatous cells to spread and migrate in early embryonic development between the ectodermal and endodermal layers [3]. MSCs are pluripotent, self-renewing, spindle-shaped cells found in several adult and perinatal tissues. To differentiate MSCs from other morphologically similar cells, the International Society for Cellular Therapy (ISCT) defined the minimal set of criteria by which MSCs are identified: adherence to plastic under normal cell culture conditions; differentiation capability into multiple cell lineages including, and not limited to, adipocytes, osteocytes, and chondrocytes; positive expression of CD105, CD73, and CD90 surface markers; and lack of expression of CD45, CD14, CD19, and CD34 and a minimal expression of HLA-DR [4]. MSCs can be derived from bone marrow (BM), adipose tissue (AT), and other adult tissues such as dental pulp and dermal tissues. MSCs can also be isolated from perinatal tissues such as cord blood (CB), placenta, and amniotic fluid and membrane (AM), as well as umbilical cord Wharton's jelly (WJ) [5-7].

MSCs possess therapeutic properties demonstrated both in vitro and in vivo, with evidence pointing to anti- 
inflammatory and immunomodulatory effects [8], as well as tissue regeneration, including healing of chronic wounds, regeneration of cartilage, angiogenesis, and vascularization following pathological conditions such as myocardial infarction, brain injury, and limb ischemia [9-14].

MSCs have generated considerable interest in the field of tissue regeneration and hold promising potential as a therapeutic approach due to their ability to enhance angiogenesis and accelerate tissue healing [15]. Indeed, angiogenesis is essential for tissue repair and an adequate vascular network is required to supply blood and growth factors to injured tissues. Although MSCs have been shown to play an important role in decreasing tissue damage and accelerating repair through the promotion of vascularization, the use of MSC-based therapies is restricted by their low level of persistence in targeted tissues and their limited capabilities of transdifferentiation in vivo [16-22]. While it was initially conceived that MSCs exerted their therapeutic effects by migrating to targeted sites of injury and actively contributed to tissue repair and regeneration, it is now increasingly acknowledged that MSCs do not typically engraft after transplantation, due to the phenomena of lung sequestration and systemic clearance $[23,24]$, and exhibit their therapeutic effect in a paracrine manner through the secretion of bioactive factors [13, 25, 26].

The paracrine effects of MSCs, firstly described by Gnecchi et al. [27], are due to numerous secreted elements collectively referred to as the secretome [28]. The secretome consists of all factors actively or passively released from cells; it contains soluble products composed of a proteic soluble fraction (mostly growth factors and cytokines) and a vesicular component, extracellular vesicles (EVs), which transfer proteins, lipids, and genetic material to recipient cells [29]. The MSC-derived secretome is very tissue- and/or individual cell-specific and is subject to fluctuations related to physiological states or pathological conditions. Moreover, the secretome is also affected by the preconditioning/priming of MSCs during cell culture prior to the collection of the conditioned media (CM) $[10,30,31]$. Thus, the appropriate therapeutic use of the MSC secretome as an active pharmaceutical ingredient as well as a drug delivery system [32] relies on the systematic quantitative and functional assessment of the MSC-secreted effectors from the perspective of specific clinical settings, e.g., macroareas such as angiogenesis, bone regeneration, and immune suppression.

In the present review, the elements of the secretome of MSCs derived from the most common tissue sources for clinical use (e.g., AT, BM, and CB) will be explored in further detail addressing their roles in the angiogenic modulation (Figure 1), and data will be compared where available.

\section{Role of Extracellular Vesicles}

One paracrine mechanism of MSCs involves the secretion of EVs that have been shown to effectively mimic the therapeutic effects of MSCs, participating in tissue repair and regeneration in several preclinical models [33-35].

EVs are a heterogeneous population of cell-derived membrane vesicles that are secreted by almost all cell types

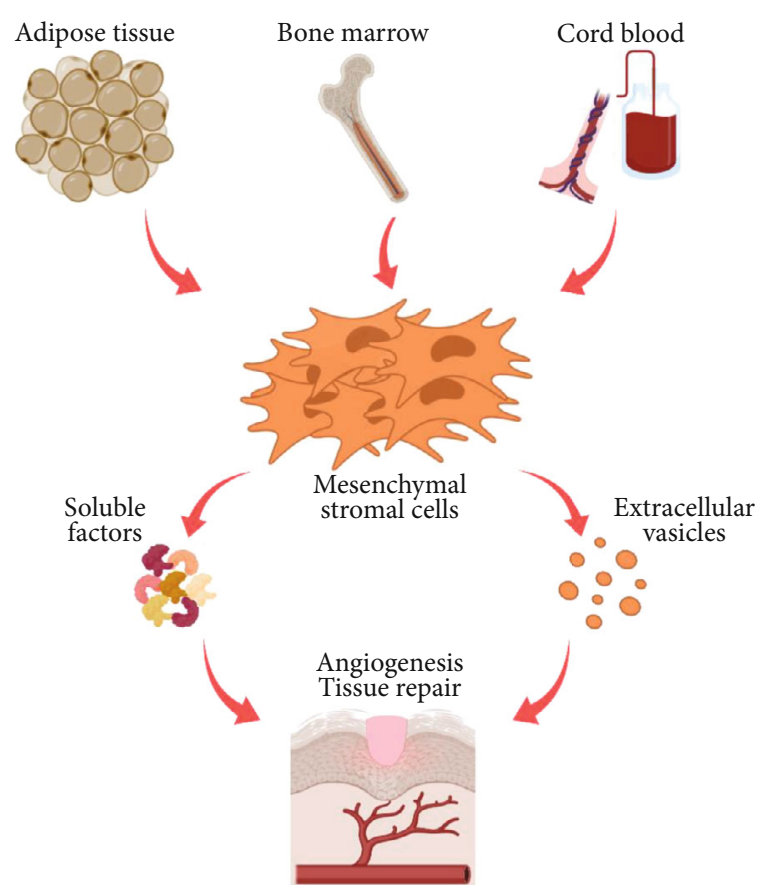

FIgURE 1: MSCs isolated and expanded from the most common sources (AT, BM, and $\mathrm{CB}$ ) release their secretome in vitro and in vivo which acts upon mechanisms responsible for enhancing tissue repair and angiogenesis.

including MSCs and serve as vehicles for bidirectional communication between cells [36]. Cells secrete a wide range of EVs that differ in size, origin, content, and function [37]. EVs include exosomes (also called small EVs), which are small membrane vesicles originating from the endocytic pathway, ranging from 30 to $150 \mathrm{~nm}$ in diameter and shed microvesicles (MVs, also called large EVs), which are large membrane vesicles of 150 to $1000 \mathrm{~nm}$ diameter budding off the plasma membrane [37]. The lipid bilayer of EVs encapsulates their bioactive contents (proteins, DNA, and RNA), protecting them from enzymatic degradation. Recently, it has become apparent that secreted EVs are proficient intercellular communication mediators through the transfer of their cargo to target cells and their ability to influence the behavior of recipient cells [36]. EVs can be purified from tissue culture supernatant as well as several biofluids (e.g., serum, plasma, saliva, ascites, cerebrospinal fluid, and urine). There is no general consensus as to the best purification method for EVs. The most common method for EV isolation is iodixanol density gradient ultracentrifugation, which separates vesicles according to their buoyant density by centrifugation. Size exclusion chromatography is also widely used for the isolation of EVs and separates vesicle particles based on their size. Immunoisolation could be a powerful method for the purification of EVs but requires the knowledge of established specific markers for EVs as well as tissue-specific discriminating markers. The International Society for Extracellular Vesicles (ISEV) has previously provided researchers with minimal experimental requirements for defining and assessing the 
quality/purity of isolated EVs in order to confidently report biological cargo or functions to EVs [38].

MSC-derived secretome performs its angiogenic modulation through a complex synergic activity between many bioactive molecules carried by EVs, such as microRNA (miRNA), transfer RNA (tRNA), long noncoding RNA (lncRNA), growth factors, proteins, and lipids [39].

2.1. EV-Associated Proteome. There is growing evidence that MSC-derived EVs play a significant role in the paracrine promotion of angiogenesis. Notably, MSCs may harbor a different angiogenic potential according to their tissue of origin. It has been reported that the AT-MSC-derived secretome displays a greater tubulogenic efficiency compared to BMMSCs due to a higher expression of angiogenic factors such as insulin growth factor- (IGF-) 1, vascular endothelial growth factor- (VEGF-) D, and interleukin- (IL-) 8 [40]. Furthermore, comparative proteomic analysis of AT-MSCderived EVs identified proteins that support a broad range of biological functions, including angiogenesis. The enriched angiogenic proteins identified in EVs comprise VEGF, von Willebrand factor (vWF), and transforming growth factor(TGF-) $\beta 1$ [41, 42]. Several functional studies investigating the paracrine actions of AT-MSCs have also involved EVs in modulating endothelial cell function and angiogenesis. For instance, it has been shown that AT-MSC-derived EVs induced in vitro vessel-like structure formation by human microvascular endothelial cells (HMEC) and that plateletderived growth factor (PDGF) stimulated the secretion of EVs and further enhanced their angiogenic potential. Indeed, compared to unstimulated cells, PDGF-stimulated MSCs release EVs carrying angiogenic c-kit, stem cell factor (SCF), and matrix metalloproteinases (MMPs) [43]. Likewise, in a skin flap ischemia/reperfusion injury model, EVs were found to increase flap recovery and capillary density (increased tube formation) in a mechanism involving IL-6 [44]. Similarly, EVs, along with AT-MSCs, were also able to protect rat kidney from acute ischemia-reperfusion injury through the induction of angiogenesis, reflected by the increase of the angiogenic factors CD31, vWF, and angiopoietin [45].

In comparison to AT-MSCs, similar angiogenic effects were observed with other sources of MSCs such as BM or CB. For instance, Bian and colleagues have previously reported that upon hypoxia stimulation, BM-MSCs release EVs that could be quickly uptaken by HUVECs promoting their proliferation and migration along with tube formation in vitro. Similar observations were made in vivo using an acute myocardial infarction rat model, where intramyocardial injection of MSC-EVs was found to markedly enhance cardiac repair [46]. Likewise, EVs from BM-MSCs were shown to increase the proliferation and migration of fibroblasts derived from normal donors and diabetic chronic wound patients as well as tube formation by endothelial cells. Mechanistically, MSC-derived EVs were found to be enriched with transcriptionally active-signal transducer and activator of transcription 3 (STAT3), which was shown to control many aspects of angiogenesis by regulating VEGF expression at the transcriptional levels [47, 48]. Moreover, analysis of proangiogenic factors from BM-MSC-derived EVs capable of inducing endothelial cell migration through extracellular signal-regulated kinase (ERK)/Akt signaling revealed the presence of high levels of extracellular matrix metalloproteinase inducer (EMMPRIN) in these vesicles [49]. EVs derived from BM-MSC were also shown to carry VEGF and to activate VEGF receptors in endothelial cells leading to enhanced angiogenesis in ischemic limbs [50]. Furthermore, wingless-related integration site 3a (Wnt3a) was also described as a cargo of a subpopulation of EVs (CD63+) capable of inducing angiogenesis in vitro [51].

Several studies have reported that hypoxia potentiates the angiogenic potency of MSC-derived EVs, whether they are originating from $\mathrm{AT}, \mathrm{BM}$, or $\mathrm{CB}$. Using a mouse model of subcutaneous fat grafting, hypoxic MSC-EVs from AT were capable of effectively promoting the survival of grafts and neovascularization (increased CD31-positive cells) [52]. Likewise, human umbilical vein endothelial cells (HUVECs) exposed to EVs from hypoxic AT-MSCs significantly upregulated angiogenesis-stimulating genes such as angiopoietin(ANG-) 1 and VEGF receptor 2 [53]. Moreover, hypoxia not only induced the expression of VEGF in AT-MSCs and their EVs but also increased VEGF expression and protein kinase A (PKA) signaling pathway in HUVECs when exposed to hypoxia EVs [53].

Indeed, EVs derived from human CB-derived MSCs stimulated by hypoxia were also shown to promote angiogenesis both in vitro and in vivo [54-57]. Mechanistically, Wnt4 was identified in EVs derived from human CB-MSCs and was shown to induce $\beta$-catenin activation in endothelial cells leading to enhanced angiogenesis [58]. More recently, a proteomic analysis identified significant enrichment of hundreds of proteins in BM-MSC-derived EVs compared to parental BM-MSCs. Among these proteins, neuropilin 1 (NRP1) is interestingly known to regulate vasculogenesis, chemotaxis, migration, and invasion [59]. Such comprehensive proteomic techniques may be used to ultimately unravel the MSCderived $\mathrm{EV}$ content and assess their angiogenic potential.

2.2. EV-Associated Nucleic Acids: miRNAs. About $98 \%$ of the entire genome is non-protein coding and was formerly termed as "junk DNA." The noncoding DNA (ncDNA) is constituted of repetitive, transposable, interspersed elements, as well as noncoding RNA (ncRNA) genes. The ncRNAs encompass about $98 \%$ of the DNA transcript. Depending on their size, ncRNAs are arbitrarily distinguished into small (sncRNAs), if composed of less than 200 nucleotides (nt) (e.g., ribosomal RNA, transfer RNA, miRNA, small interfering RNA (siRNA), and piwi-associated RNA (piRNA)), and long (lncRNAs) if they have more than $200 \mathrm{nt}$ [60]. The lncRNAs together with miRNAs, piRNAs, and endogenous siRNAs are involved in the epigenetic modification of DNA and in the regulation of transcriptional and posttranscriptional events.

In addition to growth factors and proteins, MSC-derived EVs were also reported to carry miRNAs. Since miRNAs are powerful regulators of gene expression, signaling through miRNA-EVs is a proficient paracrine mechanism used by MSCs to modulate angiogenesis. Regardless of the tissue of 
TABLE 1: Extracellular vesicle miRNA cargo and MSC-mediated angiogenesis.

\begin{tabular}{|c|c|c|c|}
\hline MicroRNA & MSC (tissue of origin) & Function & Reference \\
\hline $\begin{array}{l}\text { miR148a, miR532-5p, } \\
\text { miR378, let-7f }\end{array}$ & Porcine adipose tissue & $\begin{array}{c}\text { Putative tissue regeneration by inducing several cellular pathways } \\
\text { including angiogenesis }\end{array}$ & {$[71]$} \\
\hline miR494 & Human bone marrow & Muscle regeneration by enhancing myogenesis and angiogenesis & {$[64]$} \\
\hline miR-19a & $\begin{array}{c}\text { Rat bone marrow } \\
\text { (GATA-4-overexpressing MSC) }\end{array}$ & Cardioprotection by increasing survival and angiogenesis & {$[72,73]$} \\
\hline $\operatorname{miR}-125 \mathrm{a}$ & Human adipose tissue & Induction of angiogenesis through the repression of DLL4 expression & {$[65]$} \\
\hline miR-210 & Mouse bone marrow & $\begin{array}{l}\text { Promotion of angiogenesis in a mouse myocardial infarction model } \\
\text { through the repression of Efna3 expression in endothelial cells }\end{array}$ & {$[66,74]$} \\
\hline miR-30b & Not available & Promotion of angiogenesis in vitro and in vivo & [75] \\
\hline miR-21a-5p & Mouse bone marrow & $\begin{array}{l}\text { Cardioprotection through inhibition of proapoptotic genes; in silico } \\
\text { data support a role in angiogenesis }\end{array}$ & {$[76]$} \\
\hline $\operatorname{miR}-210-3 p$ & Mouse bone marrow & $\begin{array}{l}\text { Acceleration of recovery of hindlimb ischemia through the promotion } \\
\text { of angiogenesis }\end{array}$ & {$[50]$} \\
\hline miR-31 & Human adipose tissue & $\begin{array}{l}\text { Promotion of angiogenesis in HUVECs by targeting the } \\
\text { antiangiogenic HIF-1 gene }\end{array}$ & {$[77]$} \\
\hline $\operatorname{miR}-181 b$ & Rat adipose tissue & $\begin{array}{c}\text { Promotion of the mobility and angiogenesis of brain microvascular } \\
\text { endothelial cells (BMECs) after oxygen-glucose deprivation (OGD) } \\
\text { through the repression of TRPM7 expression }\end{array}$ & {$[63]$} \\
\hline $\operatorname{miR}-21-5 p$ & Human endometrium & $\begin{array}{l}\text { Cardioprotection and enhancing microvessel density in a rat model of } \\
\text { myocardial infarction }\end{array}$ & [78] \\
\hline
\end{tabular}

origin, enrichment of miRNAs in MSC-EVs has been shown to promote angiogenesis in vitro and in vivo (summarized in Table 1). miRNAs may act as suppressors of gene expression binding to the $3^{\prime}$-untranslated ( $3^{\prime}$-UTR) regions of specific mRNAs inhibiting their translation and/or promoting their degradation [61]. It has been reported that miRNAs target and modulate the expression of regulatory angiogenic genes encoding for cytokines, MMPs, VEGF, PDGF, fibroblast growth factor (FGF), and epidermal growth factor (EGF) [62]. For instance, miR-181b-5p was shown to modulate cerebral vascular remodeling after stroke. miR-181b-5p is carried in AT-MSC-derived EVs and enhances the mobility and angiogenesis of brain microvascular endothelial cells (BMECs) after oxygen-glucose deprivation by targeting transient receptor potential melastatin 7 (TRPM7) expression [63]. Several other miRNAs with angiogenic potential such as miRNA-494, miR-125a, or miR-210 were recently reported in MSC-derived EVs [64-66].

Moreover, MSC-EV delivery of miRNA-132, along with stromal cell-derived factor 1 (SDF-1), results in increased tube formation and enhanced angiogenic activity of endothelial cells in infarcted myocardial mouse models [67-69].

Furthermore, a recent comprehensive system study conducted by Ferguson and colleagues profiled the miRNAs contained in EVs isolated from human BM-derived MSCs and determined the dominant biological processes and pathways modulated by these miRNAs. Interestingly, around $23 \mathrm{miR}$ NAs were identified as more abundant and were found to target genes related to cardiovascular and angiogenesis processes (more than 90 genes related to vasculature and tube development were targeted by miR-23a-3p, miR-424-5p, miR-144, and miR-130a-3p; and between 9 and 85 other cardiovascular development, angiogenesis, and tube formation genes were targeted by the remaining miRNAs). Functional testing of these EVs revealed that MSC-EVs were able to protect cardiomyocytes from apoptosis and increased angiogenesis in HUVECs [70].

2.3. Other EV-Associated Nucleic Acids. Unlike the parent cell, MSC-derived-EVs are highly enriched in the class of tRNAs (more than $50 \%$ of total small RNAs in AT-derived EVs and 23-35\% in BM-derived EVs) [79]. tRNAs, along with piRNAs, contribute to maintaining stem cell potency [79], promoting cell survival and inhibiting cell differentiation of CB hematopoietic stem cells [80]. Their regulation of tissue healing and repair processes has been wellrecognized [81, 82]. Stem cells can deliver ncRNAs to injured tissues through EVs, thus regulating specific programs of tissue regeneration [83]. As an example, MSCs can differentiate into endotheliocytes and myocytes in the mesoderm under conditional induction [84]. MSC-derived lncRNAs have been shown to support angiogenesis through the endothelial differentiation of MSCs: myocardial infarction-associated transcript (MIAT) targets miR200a and VEGF [85, 86]; maternally expressed gene 3 (MEG3) facilitates the ubiquitination and degradation of forkhead box protein M1 (FOXM1) reducing the angiogenic VEGF expression and promoting endothelial differentiation of MSCs [87].

2.4. Lipids. Lipids are also an integral part of EVs, and several studies have described EV lipid composition. EVs are rich in cholesterol, phospholipid (phosphatidylserine, phosphatidylcholine, phosphatidylethanolamine, and phosphatidylinositol), sphingomyelin, glycosphingolipids, diglyceride, polyglycerol, and ganglioside GM3 [88-90]. Specific lipids are enriched in EVs compared with their parent cells. Studies have shown a 
2- to 3-fold enrichment from cells to MVs of cholesterol, glycosphingolipids, and phosphatidylserine [91-93] and a reduction of phosphatidylcholine [90].

As part of vesicle membranes, EV lipids provide the structural rigidity and stability required during the budding process as well as the ability to protect EV cargo, promoting autocrine or paracrine signaling $[89,94]$. EV uptake can also be affected by lipid composition, with lipid rafts allowing the EVs to fuse into recipient cells [89]. As a result of EVs' high lipid content, they have an inherent capacity to pass through biological barriers and escape from phagocytosis by the reticuloendothelial system, while being biocompatible and immunologically inert $[88,95]$.

EVs released from different cell sources have distinct lipid content [92]. MSC-derived EVs are enriched in long lipid species and polyunsaturated acyl chains (more than 60 carbons and 10 double bonds) [92]; lysoderivatives of some phospholipids are enriched in large EVs and cardiolipin in small EVs. These characteristics are thought to allow for the curvature required and structural arrangement [92], although any biological functions are largely unknown. EVs are known to transport many bioactive lipids as well as lipid metabolism enzymes between cells. Possible bioactive lipids contained in MSC-derived EVs include leukotrienes, arachidonic acid, phosphatidic acid, prostaglandins, lysophosphatidylcholine, and docosahexaenoic acid [96]. In addition, the fate of EVs depends on the interaction of specific receptors with vesicular phosphatidylserine and lysophosphatidylcholine [97]. These interactions allow intercellular communications [98], chemoattraction [99], and apoptosis [100]. EVs derived from human BM-MSCs show an enrichment of sphingosine-1-phosphate (S1P), which is a signaling sphingolipid mediating cell proliferation, migration, and barrier function. When applied to chondrocytes, these EVs induce proliferation, matrix deposition, and cartilage defect repair. Blocking S1P reduced the therapeutic effect of MSCderived EVs [91].

The properties of MSC-derived secretome have also been studied after cell conditioning. Human BM-MSCs cultured in the presence of omega- 6 and omega- 9 fatty acids altered MSC expression and secretion of known mediators of angiogenesis, namely, the secretion of IL-6, VEGF, and nitric oxide. This indicates that fatty acids may affect MSC engraftment to injured tissue as well as MSC secretion of cytokines and growth factors that regulate local cellular responses to injury [101]. It has also been reported that MSCs lose their multipotency and undergo senescence during prolonged in vitro culture [102] and this can partly be attributed to a decrease in omega- 6 fatty acids which in turn decreases membrane fluidity [103]. Supplementing lipids during the culture of human fetal membrane MSCs has been shown to increase cell proliferation rate, angiogenic differentiation, and immunomodulatory properties [104]. In addition, when these MSCs are exposed to EVs obtained from lipidsupplemented culture, cell migration rates improve as assessed by a wound-healing assay [105]. Depriving MSCs of serum and oxygen also alters its metabolic and lipidomic profile which in turn affects the composition of the released EVs. Under these conditions, higher ratios of lysophosphati- dylcholine and phosphatidylethanolamine as well as ceramide are reported. Importantly, these lipids are associated with lipid rafts and involved in receptor-mediated intercellular signaling, respectively [106]. Taken together, these studies highlight the central role of the membrane system and bioactive lipids in cell physiology and angiogenesis. The effects exerted by MSC-derived EVs on endothelial cells are depicted in Figure 2.

\section{Role of Soluble Proteins}

MSCs release in the extracellular space a plethora of angiogenic factors including basic fibroblast growth factor (bFGF), VEGF, transforming growth factor-beta (TGF- $\beta$ ), PDGF, ANG-1, placental growth factor (PIGF), IL-6, hepatocyte growth factor (HGF), and monocyte chemoattractant protein 1 (MCP-1), which stimulate angiogenesis in vitro and in vivo $[9,107]$. VEGF and TGF- $\beta 1$ secreted in the CM promote angiogenesis and activate PI3K/Akt and MAPK pathways $[9,108]$; HGF exhibits angiogenic properties on its own by inducing the expression of VEGF.

It has been reported throughout the literature that conditions such as exposure to tumor necrosis factor- (TNF-) $\alpha$, interferon- (IFN-) $\gamma$, or hypoxia are able to modulate the composition of the MSC-derived secretome, a method generally referred to as priming. Hypoxia and serum deprivation priming are a driving factor towards the acquisition of a proangiogenic phenotype with MSCs derived from BM, AT, and placenta. They increase the production of VEGF, bFGF, HGF, IGF, and TGF- $\beta[10,30]$ and induce lipid and metabolic modifications within the cells and secreted EVs, as described above [106]. While the effects of culture conditions and priming on MSC-derived secretome are reported, the lack of standardization and regulation regarding culturing techniques prevents the definition of ideal culturing settings for producing the most effective secretome, from the source of MSCs used, the type of culture medium and complementary serums utilized, to the use of 3D cultures and scaffolding $v s$. more traditional $2 \mathrm{D}$ cultures.

The composition and concentration of angiogenic proteins vary between different MSC sources (WJ, AT, and $\mathrm{BM})$, consequently affecting the functional responses. Recently, the angiogenic potential of WJ- and BM-derived MSCs has been described to be higher than AT-MSCs [108]. However, the paracrine activity of MSCs derived from neonatal tissues (CB and $\mathrm{AM}$ ), when compared to adult tissues (AT), is not consistent across the different sources. An unbiased stable isotope labeling by amino acid- (SILAC-) based quantitative proteomic approach in cell culture coupled to LC-MS and validated by functional assays revealed that the secretome of MSCs isolated from fetal skin is superior to that of adult skin [109]. When considered across different sources, different patterns emerge: the secretion of VEGF and TGF- $\beta 1$ is higher and comparable in AMMSCs and AT-MSCs, while CB-MSCs show lower production, with an associated opposite trend for HGF (higher secretion in CB-MSCs vs. AM-MSCs) [110, 111]. On the other hand, Kim et al. showed increased secretion of 


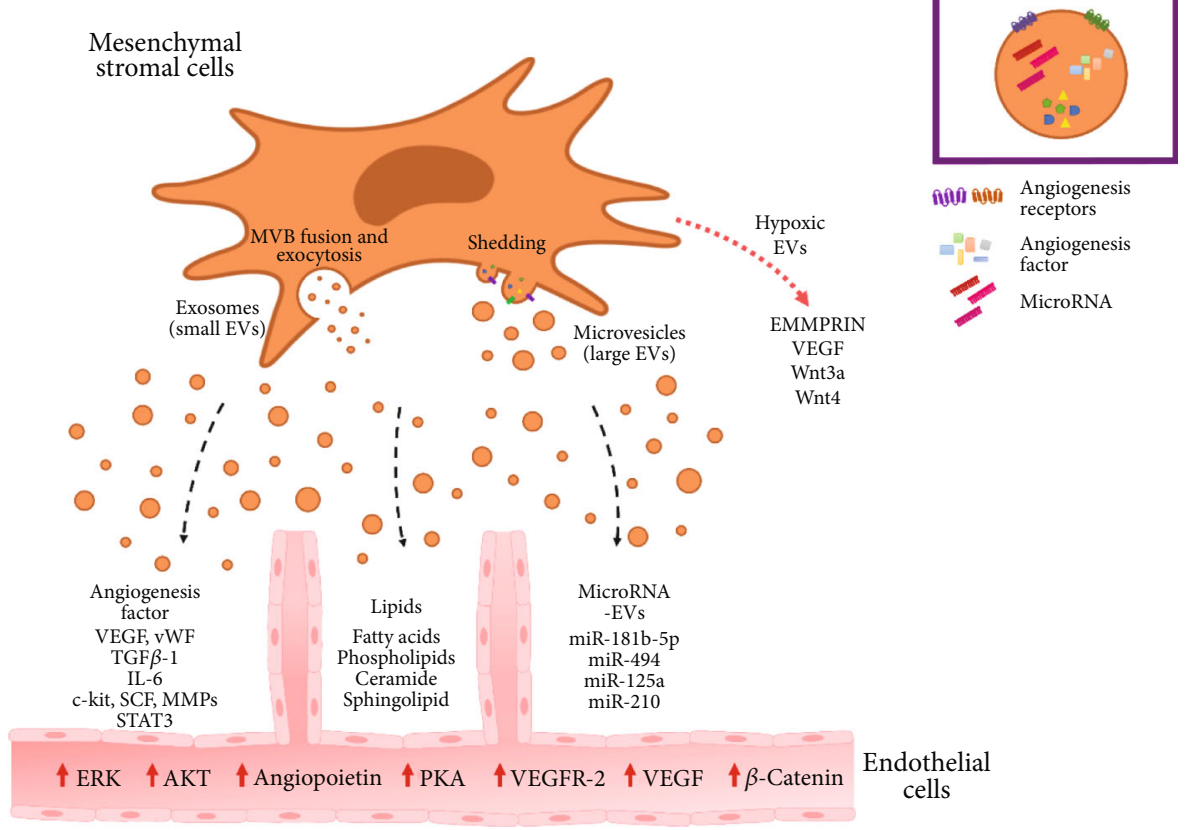

FIGURE 2: EV-mediated paracrine action of MSCs in angiogenesis. MSCs release EVs that are enriched in angiogenic factors such as cytokines, chemokines, and growth factors as well as miRNAs and lipids. Transferring of MSC-derived EV cargo to recipient endothelial cells triggers proangiogenic signaling important to tissue repair. In response to hypoxia, MSCs release EVs with an increased angiogenic potency capable of activating targeted signaling pathways to regulate the expression of angiogenic factors in endothelial cells.

VEGF-A, HGF, bFGF, and ANG-1 from amniotic-derived MSCs when compared to AT-MSCs [112].

Furthermore, macrophage colony-stimulating factor (MCSF), interleukin-1 receptor antagonist (IL-1ra), and SDF-1a secretion were significantly higher in TNF- $\alpha$ - and IFN- $\gamma$ stimulated perinatal MSCs but lower than BM-MSCs while monocyte chemotactic protein-1 (MCP-1) was significantly higher in perinatal MSCs than BM-MSCs with no changes after stimulation [111].

The reports on which MSC source yields the most potent angiogenic and tubulogenic effects are sometimes conflicting. Hsiao et al. [40] reported that AT-MSCs are superior paracrine cells when compared to dermal tissue-derived and BM-MSCs due to heightened levels of VEGF, IGF-1, and IL-8, as well as MMP-3 and MMP-9 secretion. Du and colleagues paradoxically found that the CM of BM-MSCs and placental MSCs were more proangiogenic than those of AT-MSCs and umbilical cord-derived MSCs [113].

bFGF is not consistently found in the secretome regardless of the MSC source, even after stimulation with TNF- $\alpha$ and IFN- $\gamma$ [111]. Moreover, the chemotactic factor growthrelated oncogene (GRO) has been detected at low levels in BM-MSCs but not in AT-MSCs or dermal-derived-MSCs [40]. Likewise, MCP, RANTES, SCF, and SDF-1 have shown variations in the CM of MSCs of different origins, while high levels of IL-6 are usually constantly retrieved [40].

Among all VEGF family components, VEGF-C and VEGF-D represent the major factors for angiogenesis and lymphangiogenic processes [114-116]. VEGF-D is expressed at higher levels in AT-MSCs compared to other MSCs [40] and is upregulated by hypoxia in BM-MSCs [114]. The Akt/Nrf2 pathway plays a crucial role in promoting the angiogenic-related functions of BM-MSCs [117], as demonstrated during infarction, where MSCs overexpressing AKT1 are able to preserve normal $\mathrm{pH}$ levels in the surviving myocardium [118]. FGF-2 modulates angiogenesis with autocrine mechanism [119], together with VEGF, has a potent synergistic effect on the induction of angiogenesis in vivo [120], and supports long-term angiogenic efficacy of AT-MSCs in ischemic mouse tissues [121]. A comparative MSC-derived secretome profile relevant to angiogenesis is provided in Table 2 . Finally, the angiogenic activity mediated by the secretome can be significantly inhibited by neutralizing antibodies against specific cytokines, such as VEGF, MCP-1, and IL-6 [9]. The MSC-derived secretome therapeutic potential to accelerate angiogenesis is extensively reviewed by Bronckaers et al. [122].

In addition to antibody-based techniques assessing known angiogenic factors present in the secretome of MSC, the field is in need of more exhaustive proteomic techniques. Indeed, a comprehensive analysis of the MSC-derived secretome is an essential step to a better understanding of the therapeutic components of MSCs. To date, mass spectrometry represents a powerful high-throughput technique enabling the identification and quantification of thousands of proteins present in the MSC secretome [108, 123, 124]. Taking advantage of such high-throughput proteomic techniques will certainly improve the characterization of MSC secretome and help to judiciously implement MSC-based therapeutic products in the clinic. 
TABle 2: Angiogenic secretome of MSCs from different sources.

\begin{tabular}{|c|c|c|c|c|}
\hline Angiogenic factors & High & Low & None & Ref \\
\hline Angiogenic potential & WJ-MSC, BM-MSC, placenta-MSC & AT-MSC, umbilical cord & & $\begin{array}{l}{[108]} \\
{[114]}\end{array}$ \\
\hline VEGF secretion & AM-MSC, AT-MSC & CB-MSC, BM-MSC & & [112] [40] \\
\hline TGF- $\beta 1$ secretion & AM-MSC & AT-MSC & & [112] \\
\hline VEGF-A, HGF, bFGF, ANG-1 & AM-MSC & AT-MSC & & [113] \\
\hline M-CSF, IL-1ra, SDF- $1 \alpha$ & Primed perinatal MSCs & BM-MSCs & & {$[112]$} \\
\hline MCP-1 & Perinatal MSCs & BM-MSCs & & {$[112]$} \\
\hline IGF-1, IL-8, MMP-3, MMP-9 & AT-MSC & BM-MSC, D-MSC & & {$[40]$} \\
\hline GRO & & BM-MSCs & AT-MSCs, D-MSCs & [40] \\
\hline IL-6 & MSCs & & & [9] [108] \\
\hline VEGF-D & AT-MSC, BM-MSCs & & & {$[40,113]$} \\
\hline FGF-2 & AT-MSC & & & [120] \\
\hline
\end{tabular}

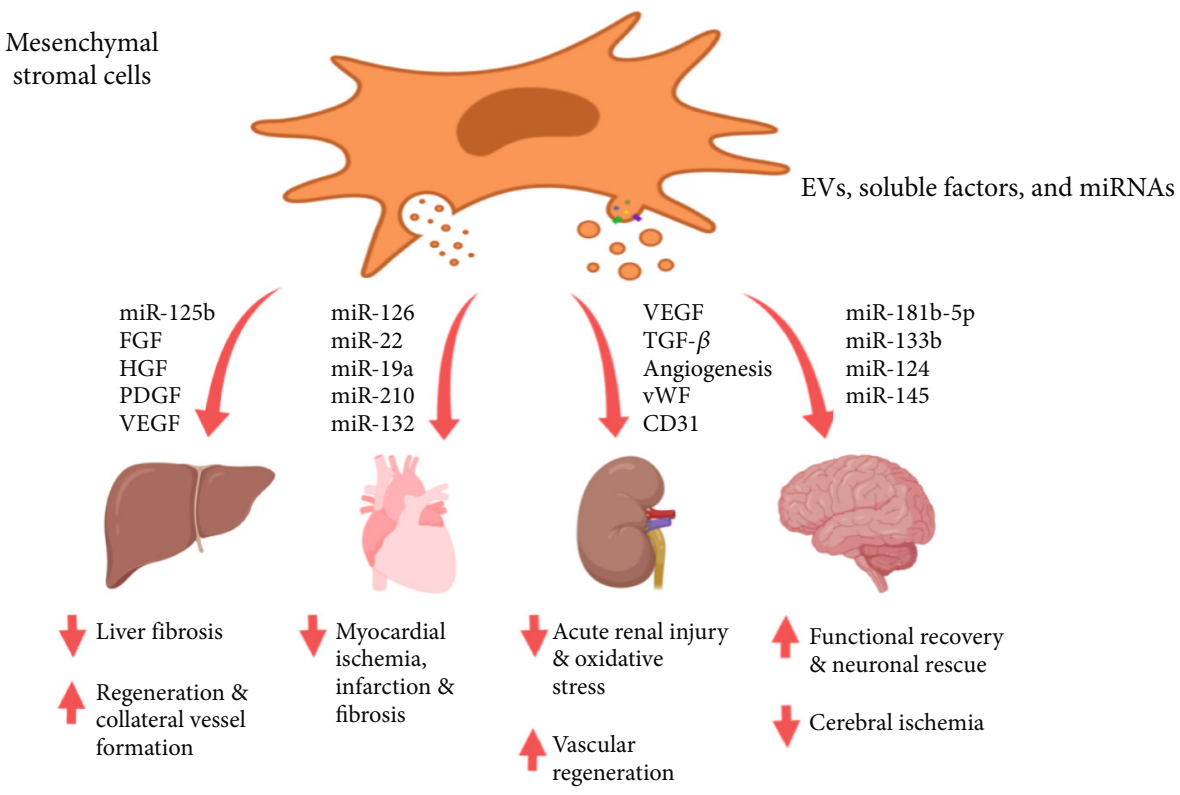

FIGURE 3: Schematic representation of the secretome effects on major organs in vivo. EVs, soluble factors, proteins, and miRNAs present in the MSC secretome are in favor of regeneration, reperfusion, and recovery of major organs and tissues following trauma and ischemic injury.

\section{Limited Clinical Studies}

The MSC secretome represents a promising angiogenic therapy option for treating ischemic diseases (e.g., coronary vascular disease, cerebral infarction, and limb ischemia), neurodegeneration, wound healing, tissue/organ fibrosis, etc. [125] (Figure 3 provides schematic representation). However, until now, it has only been studied and applied in experimental models, though numerous.

The sole clinical trial currently registered on http:// clinicaltrial.gov/ is the NCT03384433 for the use of allogenic MSC-small EVs in patients with acute ischemic stroke, which however did not recruit patients so far. Nonetheless, one limited clinical report has been published in a different clinical setting (refractory graft versus host disease) [126].
Challenges and constraints in clinical-grade production of MSC secretome, choice of MSC source (likely to be specifically addressed per each disease setting), and preconditioning have been recently reviewed by Teixeira and Salgado [127].

\section{Conclusions}

Therapeutic angiogenesis depends on the efficient delivery of exogenous angiogenic factors to stimulate neovasculature formation. MSC-derived secretome seems to mediate beneficial angiogenic effects in a variety of tissue injury-related diseases, therefore supporting the development of cell-free therapeutic products that exert similar desirable effects to systemic MSC infusion therapy. Interestingly, MSC-derived 
secretome as a cell-free therapy bypasses the risks associated with stem cell-based therapies, namely, immune-mediated rejection, accumulation of genomic alterations, senescenceinduced genetic instability, and complicated safety regulatory setting [128-131].

The expected successful clinical application of MSCderived secretome will require a comprehensive understanding of its different components, the in vivo functionality of the proteins secreted, and their specificity to a given pathological setting. Definitely, a major cause of failure of MSCbased therapeutic products is the incomplete characterization of their features and activities. The successful clinical application of MSC-secreted factors will require many more comprehensive studies using an "omics" approach, including quantitative proteomic assessment of different MSC populations of clinical relevance. Although comprehensive and quantitative information on proteins through mass spectrometry can appear more challenging (compared to gene expression or antibody-based assays), high-throughput proteomic techniques are greatly needed for deciphering the protein make-up of MSCs as well as quantifying the changes in physiological or pathological conditions. New technological and bioinformatics advances in mass spectrometry bring this closer to reality [132]. Moreover, the development of standardized procedures for large-scale clinical-grade secretome-based products and their manufacturing, which is highly needed for future clinical trials, remains insufficient. Therefore, we believe that the current resources dedicated to MSC-related regenerative therapies should be better exploited to unveil the features and functionality of the MSC secretome and to enable a GMP-standardized production of ready-off-the-shelf, well-characterized acellular products. This investment is indeed more likely to pay back by providing a stable therapeutic strategy which is well characterized than using MSCs as cell products.

\section{Conflicts of Interest}

The authors declare that there is no conflict of interest regarding the publication of this paper.

\section{Acknowledgments}

The authors acknowledge Dr. Ahmed Makki (Plastic Surgicentre, Doha) for continuously supporting the Mesenchymal Stromal Cell Project at Sidra Medicine. This manuscript was funded by a National Priorities Research Program (NPRP12S-0304-190225) grant from the Qatar National Research Fund (QNRF).

\section{References}

[1] A. J. Friedenstein, R. K. Chailakhjan, and K. S. Lalykina, “The development of fibroblast colonies in monolayer cultures of guinea-pig bone marrow and spleen cells," Cell and Tissue Kinetics, vol. 3, no. 4, pp. 393-403, 1970.

[2] A. J. Friedenstein, R. K. Chailakhyan, N. V. Latsinik, A. F. Panasyuk, and I. V. Keiliss-Borok, "Stromal cells responsible for transferring the microenvironment of the hemopoietic tissues. Cloning in vitro and retransplantation in vivo," Transplantation, vol. 17, no. 4, pp. 331-340, 1974.

[3] A. I. Caplan, "Mesenchymal stem cells," Journal of Orthopaedic Research, vol. 9, no. 5, pp. 641-650, 1991.

[4] M. Dominici, K. le Blanc, I. Mueller et al., "Minimal criteria for defining multipotent mesenchymal stromal cells. The International Society for Cellular Therapy position statement," Cytotherapy, vol. 8, no. 4, pp. 315-317, 2006.

[5] M. F. Pittenger, A. M. Mackay, S. C. Beck et al., "Multilineage potential of adult human mesenchymal stem cells," Science, vol. 284, no. 5411, pp. 143-147, 1999.

[6] R. Hass, C. Kasper, S. Böhm, and R. Jacobs, "Different populations and sources of human mesenchymal stem cells (MSC): a comparison of adult and neonatal tissue-derived MSC," Cell Communication and Signaling: CCS, vol. 9, no. 1, 2011.

[7] L. A. Marquez-Curtis, A. Janowska-Wieczorek, L. E. McGann, and J. A. W. Elliott, "Mesenchymal stromal cells derived from various tissues: biological, clinical and cryopreservation aspects," Cryobiology, vol. 71, no. 2, pp. 181-197, 2015.

[8] M. E. Bernardo and W. E. Fibbe, "Mesenchymal stromal cells: sensors and switchers of inflammation," Cell Stem Cell, vol. 13, no. 4, pp. 392-402, 2013.

[9] H. M. Kwon, S. M. Hur, K. Y. Park et al., "Multiple paracrine factors secreted by mesenchymal stem cells contribute to angiogenesis," Vascular Pharmacology, vol. 63, no. 1, pp. 19-28, 2014.

[10] C. J. Cunningham, E. Redondo-Castro, and S. M. Allan, "The therapeutic potential of the mesenchymal stem cell secretome in ischaemic stroke," Journal of Cerebral Blood Flow and Metabolism, vol. 38, no. 8, pp. 1276-1292, 2018.

[11] C. T. J. van Velthoven, A. Kavelaars, and C. J. Heijnen, "Mesenchymal stem cells as a treatment for neonatal ischemic brain damage," Pediatric Research, vol. 71, no. 2-4, pp. 474481, 2012.

[12] T. Iwase, N. Nagaya, T. Fujii et al., "Comparison of angiogenic potency between mesenchymal stem cells and mononuclear cells in a rat model of hindlimb ischemia," Cardiovascular Research, vol. 66, no. 3, pp. 543-551, 2005.

[13] R. H. Lee, A. A. Pulin, M. J. Seo et al., "Intravenous hMSCs improve myocardial infarction in mice because cells embolized in lung are activated to secrete the anti-inflammatory protein TSG-6," Cell Stem Cell, vol. 5, no. 1, pp. 54-63, 2009.

[14] S. A. Muhammad, "Mesenchymal stromal cell secretome as a therapeutic strategy for traumatic brain injury," BioFactors, vol. 45, no. 6, pp. 880-891, 2019.

[15] A. I. Caplan and D. Correa, "The MSC: an injury drugstore," Cell Stem Cell, vol. 9, no. 1, pp. 11-15, 2011.

[16] R. Z. Lin, R. Moreno-Luna, B. Zhou, W. T. Pu, and J. M. Melero-Martin, "Equal modulation of endothelial cell function by four distinct tissue-specific mesenchymal stem cells," Angiogenesis, vol. 15, no. 3, pp. 443-455, 2012.

[17] S. Merfeld-Clauss, N. Gollahalli, K. L. March, and D. O. Traktuev, "Adipose tissue progenitor cells directly interact with endothelial cells to induce vascular network formation," Tissue Engineering. Part A, vol. 16, no. 9, pp. 2953-2966, 2010.

[18] S. Kachgal and A. J. Putnam, "Mesenchymal stem cells from adipose and bone marrow promote angiogenesis via distinct cytokine and protease expression mechanisms," Angiogenesis, vol. 14, no. 1, pp. 47-59, 2011. 
[19] D. O. Traktuev, S. Merfeld-Clauss, J. Li et al., "A population of multipotent CD34-positive adipose stromal cells share pericyte and mesenchymal surface markers, reside in a periendothelial location, and stabilize endothelial networks," Circulation Research, vol. 102, no. 1, pp. 77-85, 2008.

[20] C. Toma, W. R. Wagner, S. Bowry, A. Schwartz, and F. Villanueva, "Fate of culture-expanded mesenchymal stem cells in the microvasculature: in vivo observations of cell kinetics," Circulation Research, vol. 104, no. 3, pp. 398-402, 2009.

[21] C. E. Murry, M. H. Soonpaa, H. Reinecke et al., "Haematopoietic stem cells do not transdifferentiate into cardiac myocytes in myocardial infarcts," Nature, vol. 428, no. 6983, pp. 664-668, 2004.

[22] M. Leiker, G. Suzuki, V. S. Iyer, Canty JMJr., and T. Lee, "Assessment of a nuclear affinity labeling method for tracking implanted mesenchymal stem cells," Cell Transplantation, vol. 17, no. 8, pp. 911-922, 2008.

[23] J. A. Ankrum, J. F. Ong, and J. M. Karp, "Mesenchymal stem cells: immune evasive, not immune privileged," Nature Biotechnology, vol. 32, no. 3, pp. 252-260, 2014.

[24] A. Galleu, Y. Riffo-Vasquez, C. Trento et al., "Apoptosis in mesenchymal stromal cells induces in vivo recipientmediated immunomodulation," Science Translational Medicine, vol. 9, no. 416, p. eaam7828, 2017.

[25] F. Tögel, Z. Hu, K. Weiss, J. Isaac, C. Lange, and C. Westenfelder, "Administered mesenchymal stem cells protect against ischemic acute renal failure through differentiation-independent mechanisms," American Journal of Physiology. Renal Physiology, vol. 289, no. 1, pp. F31-F42, 2005.

[26] A. I. Caplan and J. E. Dennis, "Mesenchymal stem cells as trophic mediators," Journal of Cellular Biochemistry, vol. 98, no. 5, pp. 1076-1084, 2006.

[27] M. Gnecchi, H. He, N. Noiseux et al., "Evidence supporting paracrine hypothesis for Akt-modified mesenchymal stem cell-mediated cardiac protection and functional improvement," The FASEB Journal, vol. 20, no. 6, pp. 661-669, 2006.

[28] F. D. Liu, K. Tam, N. Pishesha, Z. Poon, and K. van Vliet, "Improving hematopoietic recovery through modeling and modulation of the mesenchymal stromal cell secretome," Stem Cell Research \& Therapy, vol. 9, no. 1, p. 268, 2018.

[29] L. Beer, M. Mildner, and H. J. Ankersmit, "Cell secretome based drug substances in regenerative medicine: when regulatory affairs meet basic science," Annals of Translational Medicine, vol. 5, no. 7, p. 170, 2017.

[30] L. Liu, J. Gao, Y. Yuan, Q. Chang, Y. Liao, and F. Lu, "Hypoxia preconditioned human adipose derived mesenchymal stem cells enhance angiogenic potential via secretion of increased VEGF and bFGF," Cell Biology International, vol. 37, no. 6, pp. 551-560, 2013.

[31] R. Tasso, M. Gaetani, E. Molino et al., "The role of bFGF on the ability of MSC to activate endogenous regenerative mechanisms in an ectopic bone formation model," Biomaterials, vol. 33, no. 7, pp. 2086-2096, 2012.

[32] E. Bari, S. Perteghella, D. di Silvestre et al., "Pilot production of mesenchymal stem/stromal freeze-dried secretome for cellfree regenerative nanomedicine: a validated GMP-compliant process," Cells, vol. 7, no. 11, p. 190, 2018.

[33] P. Goodarzi, B. Larijani, S. Alavi-Moghadam et al., "Mesenchymal stem cells-derived exosomes for wound regenera- tion," Advances in Experimental Medicine and Biology, vol. 1119, pp. 119-131, 2018.

[34] D. G. Phinney and M. F. Pittenger, "Concise review: MSCderived exosomes for cell-free therapy," Stem Cells, vol. 35, no. 4, pp. 851-858, 2017.

[35] R. C. Lai, F. Arslan, M. M. Lee et al., "Exosome secreted by MSC reduces myocardial ischemia/reperfusion injury," Stem Cell Research, vol. 4, no. 3, pp. 214-222, 2010.

[36] M. Tkach and C. Thery, "Communication by extracellular vesicles: where we are and where we need to go," Cell, vol. 164, no. 6, pp. 1226-1232, 2016.

[37] M. Colombo, G. Raposo, and C. Thery, "Biogenesis, secretion, and intercellular interactions of exosomes and other extracellular vesicles," Annual Review of Cell and Developmental Biology, vol. 30, pp. 255-289, 2014.

[38] C. Théry, K. W. Witwer, E. Aikawa et al., "Minimal information for studies of extracellular vesicles 2018 (MISEV2018): a position statement of the International Society for Extracellular Vesicles and update of the MISEV2014 guidelines," Journal of Extracellular Vesicles, vol. 7, no. 1, p. 1535750, 2018.

[39] S. Keerthikumar, D. Chisanga, D. Ariyaratne et al., "ExoCarta: a web-based compendium of exosomal cargo," Journal of Molecular Biology, vol. 428, no. 4, pp. 688-692, 2016.

[40] S. T. Hsiao, A. Asgari, Z. Lokmic et al., "Comparative analysis of paracrine factor expression in human adult mesenchymal stem cells derived from bone marrow, adipose, and dermal tissue," Stem Cells and Development, vol. 21, no. 12, pp. 2189-2203, 2012.

[41] A. Eirin, X. Y. Zhu, A. S. Puranik et al., "Comparative proteomic analysis of extracellular vesicles isolated from porcine adipose tissue-derived mesenchymal stem/stromal cells," Scientific Reports, vol. 6, no. 1, 2016.

[42] A. Eirin, X. Y. Zhu, A. S. Puranik et al., "Integrated transcriptomic and proteomic analysis of the molecular cargo of extracellular vesicles derived from porcine adipose tissue-derived mesenchymal stem cells," PLoS One, vol. 12, no. 3, p. e0174303, 2017.

[43] T. Lopatina, S. Bruno, C. Tetta, N. Kalinina, M. Porta, and G. Camussi, "Platelet-derived growth factor regulates the secretion of extracellular vesicles by adipose mesenchymal stem cells and enhances their angiogenic potential," Cell Communication and Signaling: CCS, vol. 12, no. 1, p. 26, 2014.

[44] C. M. Pu, C. W. Liu, C. J. Liang et al., “Adipose-derived stem cells protect skin flaps against ischemia/reperfusion injury via IL-6 expression," The Journal of Investigative Dermatology, vol. 137, no. 6, pp. 1353-1362, 2017.

[45] K. C. Lin, H. K. Yip, P. L. Shao et al., "Combination of adipose-derived mesenchymal stem cells (ADMSC) and ADMSC-derived exosomes for protecting kidney from acute ischemia-reperfusion injury," International Journal of Cardiology, vol. 216, pp. 173-185, 2016.

[46] S. Bian, L. Zhang, L. Duan, X. Wang, Y. Min, and H. Yu, "Extracellular vesicles derived from human bone marrow mesenchymal stem cells promote angiogenesis in a rat myocardial infarction model," Journal of Molecular Medicine (Berlin, Germany), vol. 92, no. 4, pp. 387-397, 2014.

[47] A. Shabbir, A. Cox, L. Rodriguez-Menocal, M. Salgado, and E. van Badiavas, "Mesenchymal stem cell exosomes induce proliferation and migration of normal and chronic wound 
fibroblasts, and enhance angiogenesis in vitro," Stem Cells and Development, vol. 24, no. 14, pp. 1635-1647, 2015.

[48] Z. Chen and Z. C. Han, "STAT3: a critical transcription activator in angiogenesis," Medicinal Research Reviews, vol. 28, no. 2, pp. 185-200, 2008.

[49] K. R. Vrijsen, J. A. Maring, S. A. Chamuleau et al., "Exosomes from cardiomyocyte progenitor cells and mesenchymal stem cells stimulate angiogenesis via EMMPRIN," Advanced Healthcare Materials, vol. 5, no. 19, pp. 2555-2565, 2016.

[50] P. Gangadaran, R. L. Rajendran, H. W. Lee et al., "Extracellular vesicles from mesenchymal stem cells activates VEGF receptors and accelerates recovery of hindlimb ischemia," Journal of Controlled Release, vol. 264, pp. 112-126, 2017.

[51] J. D. McBride, L. Rodriguez-Menocal, W. Guzman, A. Candanedo, M. Garcia-Contreras, and E. V. Badiavas, "Bone marrow mesenchymal stem cell-derived CD63+ exosomes transport Wnt3a exteriorly and enhance dermal fibroblast proliferation, migration, and angiogenesis in vitro," Stem Cells and Development, vol. 26, no. 19, pp. 1384-1398, 2017.

[52] Y. D. Han, Y. Bai, X. L. Yan et al., "Co-transplantation of exosomes derived from hypoxia-preconditioned adipose mesenchymal stem cells promotes neovascularization and graft survival in fat grafting," Biochemical and Biophysical Research Communications, vol. 497, no. 1, pp. 305-312, 2018.

[53] C. Xue, Y. Shen, X. Li et al., "Exosomes derived from hypoxiatreated human adipose mesenchymal stem cells enhance angiogenesis through the PKA signaling pathway," Stem Cells and Development, vol. 27, no. 7, pp. 456-465, 2018.

[54] H. C. Zhang, X. B. Liu, S. Huang et al., "Microvesicles derived from human umbilical cord mesenchymal stem cells stimulated by hypoxia promote angiogenesis both in vitro and in vivo," Stem Cells and Development, vol. 21, no. 18, pp. 3289-3297, 2012.

[55] C. Salomon, J. Ryan, L. Sobrevia et al., "Exosomal signaling during hypoxia mediates microvascular endothelial cell migration and vasculogenesis," PLoS One, vol. 8, no. 7, p. e68451, 2013.

[56] M. Komaki, Y. Numata, C. Morioka et al., "Exosomes of human placenta-derived mesenchymal stem cells stimulate angiogenesis," Stem Cell Research \& Therapy, vol. 8, no. 1, p. 219, 2017.

[57] Y. Zhao, X. Sun, W. Cao et al., "Exosomes derived from human umbilical cord mesenchymal stem cells relieve acute myocardial ischemic injury," Stem Cells International, vol. 2015, 12 pages, 2015.

[58] B. Zhang, X. Wu, X. Zhang et al., "Human umbilical cord mesenchymal stem cell exosomes enhance angiogenesis through the Wnt $4 / \beta$-catenin pathway," Stem Cells Translational Medicine, vol. 4, no. 5, pp. 513-522, 2015.

[59] A. Munshi, J. Mehic, M. Creskey et al., "A comprehensive proteomics profiling identifies NRP1 as a novel identity marker of human bone marrow mesenchymal stromal cellderived small extracellular vesicles," Stem Cell Research \& Therapy, vol. 10, no. 1, p. 401, 2019.

[60] T. R. Cech and J. A. Steitz, "The noncoding RNA revolutiontrashing old rules to forge new ones," Cell, vol. 157, no. 1, pp. 77-94, 2014.

[61] T. Ma, Y. Chen, Y. Chen et al., "MicroRNA-132, Delivered by Mesenchymal Stem Cell-Derived Exosomes, Promote Angio- genesis in Myocardial Infarction," Stem Cells International, vol. 2018, Article ID 3290372, 11 pages, 2018.

[62] Y. M. Salinas-Vera, L. A. Marchat, D. Gallardo-Rincón et al., "AngiomiRs: microRNAs driving angiogenesis in cancer (review)," International Journal of Molecular Medicine, vol. 43, no. 2, pp. 657-670, 2019.

[63] Y. Yang, Y. Cai, Y. Zhang, J. Liu, and Z. Xu, "Exosomes secreted by adipose-derived stem cells contribute to angiogenesis of brain microvascular endothelial cells following oxygen-glucose deprivation in vitro through microRNA181b/TRPM7 axis," Journal of Molecular Neuroscience, vol. 65, no. 1, pp. 74-83, 2018.

[64] Y. Nakamura, S. Miyaki, H. Ishitobi et al., "Mesenchymalstem-cell-derived exosomes accelerate skeletal muscle regeneration," FEBS Letters, vol. 589, no. 11, pp. 1257-1265, 2015.

[65] X. Liang, L. Zhang, S. Wang, Q. Han, and R. C. Zhao, "Exosomes secreted by mesenchymal stem cells promote endothelial cell angiogenesis by transferring miR-125a," Journal of Cell Science, vol. 129, no. 11, pp. 2182-2189, 2016.

[66] C. T. Lin, C. H. Wang, K. W. Ou et al., "Clinical applications of the pedicled anterolateral thigh flap in reconstruction," ANZ Journal of Surgery, vol. 87, no. 6, pp. 499-504, 2017.

[67] X. H. Gong, H. Liu, S. J. Wang, S. W. Liang, and G. G. Wang, "Exosomes derived from SDF1-overexpressing mesenchymal stem cells inhibit ischemic myocardial cell apoptosis and promote cardiac endothelial microvascular regeneration in mice with myocardial infarction," Journal of Cellular Physiology, vol. 234, no. 8, pp. 13878-13893, 2018.

[68] C. R. Harrell, C. Fellabaum, N. Jovicic, V. Djonov, N. Arsenijevic, and V. Volarevic, "Molecular mechanisms responsible for therapeutic potential of mesenchymal stem cell-derived secretome," Cell, vol. 8, no. 5, p. 467, 2019.

[69] C. Ju, Y. Shen, G. Ma et al., "Transplantation of cardiac mesenchymal stem cell-derived exosomes promotes repair in ischemic myocardium," Journal of Cardiovascular Translational Research, vol. 11, no. 5, pp. 420-428, 2018.

[70] S. W. Ferguson, J. Wang, C. J. Lee et al., "The microRNA regulatory landscape of MSC-derived exosomes: a systems view," Scientific Reports, vol. 8, no. 1, p. 1419, 2018.

[71] A. Eirin, S. M. Riester, X. Y. Zhu et al., "MicroRNA and mRNA cargo of extracellular vesicles from porcine adipose tissue-derived mesenchymal stem cells," Gene, vol. 551, no. 1, pp. 55-64, 2014.

[72] H. Li, S. Zuo, Z. He et al., "Paracrine factors released by GATA-4 overexpressed mesenchymal stem cells increase angiogenesis and cell survival," American Journal of Physiology. Heart and Circulatory Physiology, vol. 299, no. 6, pp. H1772-H1781, 2010.

[73] B. Yu, H. W. Kim, M. Gong et al., "Exosomes secreted from GATA-4 overexpressing mesenchymal stem cells serve as a reservoir of anti-apoptotic microRNAs for cardioprotection," International Journal of Cardiology, vol. 182, pp. 349-360, 2015.

[74] G. J. Moon, J. H. Sung, D. H. Kim et al., “Application of mesenchymal stem cell-derived extracellular vesicles for stroke: biodistribution and microRNA study," Translational Stroke Research, vol. 10, no. 5, pp. 509-521, 2019.

[75] M. Gong, B. Yu, J. Wang et al., "Mesenchymal stem cells release exosomes that transfer miRNAs to endothelial cells and promote angiogenesis," Oncotarget, vol. 8, no. 28, pp. 45200-45212, 2017. 
[76] K. M. Luther, L. Haar, M. McGuinness et al., "Exosomal miR21a-5p mediates cardioprotection by mesenchymal stem cells," Journal of Molecular and Cellular Cardiology, vol. 119, pp. 125-137, 2018.

[77] T. Kang, T. M. Jones, C. Naddell et al., “Adipose-derived stem cells induce angiogenesis via microvesicle transport of miRNA-31," Stem Cells Translational Medicine, vol. 5, no. 4, pp. 440-450, 2016.

[78] K. Wang, Z. Jiang, K. A. Webster et al., "Enhanced cardioprotection by human endometrium mesenchymal stem cells driven by exosomal microRNA-21," Stem Cells Translational Medicine, vol. 6, no. 1, pp. 209-222, 2017.

[79] S. R. Baglio, K. Rooijers, D. Koppers-Lalic et al., "Human bone marrow- and adipose-mesenchymal stem cells secrete exosomes enriched in distinctive miRNA and tRNA species," Stem Cell Research \& Therapy, vol. 6, no. 1, 2015.

[80] L. de Luca, S. Trino, I. Laurenzana et al., "MiRNAs and piRNAs from bone marrow mesenchymal stem cell extracellular vesicles induce cell survival and inhibit cell differentiation of cord blood hematopoietic stem cells: a new insight in transplantation," Oncotarget, vol. 7, no. 6, pp. 6676-6692, 2016.

[81] S. Ounzain, S. Crippa, and T. Pedrazzini, "Small and long non-coding RNAs in cardiac homeostasis and regeneration," Biochimica et Biophysica Acta, vol. 1833, no. 4, pp. 923-933, 2013.

[82] S. Ounzain and T. Pedrazzini, "The promise of enhancerassociated long noncoding RNAs in cardiac regeneration," Trends in Cardiovascular Medicine, vol. 25, no. 7, pp. 592602, 2015.

[83] F. Fatima and M. Nawaz, "Stem cell-derived exosomes: roles in stromal remodeling, tumor progression, and cancer immunotherapy," Chinese Journal of Cancer, vol. 34, no. 12, pp. 541-553, 2015.

[84] A. Uccelli, L. Moretta, and V. Pistoia, "Mesenchymal stem cells in health and disease," Nature Reviews. Immunology, vol. 8, no. 9, pp. 726-736, 2008.

[85] C. Sun, L. Huang, Z. Li et al., "Long non-coding RNA MIAT in development and disease: a new player in an old game," Journal of Biomedical Science, vol. 25, no. 1, p. 23, 2018.

[86] H. Wang, X. G. Ding, J. J. Yang et al., "LncRNA MIAT facilitated BM-MSCs differentiation into endothelial cells and restored erectile dysfunction via targeting miR-200a in a rat model of erectile dysfunction," European Journal of Cell Biology, vol. 97, no. 3, pp. 180-189, 2018.

[87] X. Sun, L. H. Luo, L. Feng, and D. S. Li, "Down-regulation of lncRNA MEG3 promotes endothelial differentiation of bone marrow derived mesenchymal stem cells in repairing erectile dysfunction," Life Sciences, vol. 208, pp. 246-252, 2018.

[88] X. Bian, K. Ma, C. Zhang, and X. Fu, "Therapeutic angiogenesis using stem cell-derived extracellular vesicles: an emerging approach for treatment of ischemic diseases," Stem Cell Research \& Therapy, vol. 10, no. 1, p. 158, 2019.

[89] M. Colombo, D. Giannandrea, E. Lesma, A. Basile, and R. Chiaramonte, "Extracellular vesicles enhance multiple myeloma metastatic dissemination," International Journal of Molecular Sciences, vol. 20, no. 13, p. 3236, 2019.

[90] D.-S. Choi, D.-K. Kim, Y.-K. Kim, and Y. S. Gho, "Proteomics, transcriptomics and lipidomics of exosomes and ectosomes," Proteomics, vol. 13, no. 10-11, pp. 1554-1571, 2013.

[91] C. Xiang, K. Yang, Z. Liang et al., "Sphingosine-1-phosphate mediates the therapeutic effects of bone marrow mesenchy- mal stem cell-derived microvesicles on articular cartilage defect," Translational Research, vol. 193, pp. 42-53, 2018.

[92] R. A. Haraszti, M.-C. Didiot, E. Sapp et al., "High-resolution proteomic and lipidomic analysis of exosomes and microvesicles from different cell sources," Journal of Extracellular Vesicles, vol. 5, no. 1, p. 32570, 2016.

[93] A. Llorente, T. Skotland, T. Sylvänne et al., "Molecular lipidomics of exosomes released by PC-3 prostate cancer cells," Biochimica et Biophysica Acta (BBA) - Molecular and Cell Biology of Lipids, vol. 1831, no. 7, pp. 1302-1309, 2013.

[94] M. Record, K. Carayon, M. Poirot, and S. Silvente-Poirot, "Exosomes as new vesicular lipid transporters involved in cell-cell communication and various pathophysiologies," Biochimica et Biophysica Acta, vol. 1841, no. 1, pp. 108-120, 2014.

[95] F. Shahabipour, M. Banach, and A. Sahebkar, "Exosomes as nanocarriers for siRNA delivery: paradigms and challenges," Archives of Medical Science, vol. 12, no. 6, pp. 1324-1326, 2016.

[96] H. Deng, C. Sun, Y. Sun et al., "Lipid, protein, and microRNA composition within mesenchymal stem cell-derived exosomes," Cellular Reprogramming, vol. 20, no. 3, pp. 178$186,2018$.

[97] M. Miyanishi, K. Tada, M. Koike, Y. Uchiyama, T. Kitamura, and S. Nagata, "Identification of Tim4 as a phosphatidylserine receptor," Nature, vol. 450, no. 7168, pp. 435-439, 2007.

[98] G. J. Freeman, J. M. Casasnovas, D. T. Umetsu, and R. H. DeKruyff, "TIMgenes: a family of cell surface phosphatidylserine receptors that regulate innate and adaptive immunity," Immunological Reviews, vol. 235, no. 1, pp. 172-189, 2010.

[99] C. G. Radu, L. V. Yang, M. Riedinger, M. Au, and O. N. Witte, "T cell chemotaxis to lysophosphatidylcholine through the G2A receptor," Proceedings of the National Academy of Sciences, vol. 101, no. 1, pp. 245-250, 2004.

[100] L. Blanc, C. Barres, P. Bette-Bobillo, and M. Vidal, "Reticulocyte-secreted exosomes bind natural IgM antibodies: involvement of a ROS-activatable endosomal phospholipase iPLA2," Blood, vol. 110, no. 9, pp. 3407-3416, 2007.

[101] A. N. Smith, L. A. Muffley, A. N. Bell, S. Numhom, and A. M. Hocking, "Unsaturated fatty acids induce mesenchymal stem cells to increase secretion of angiogenic mediators," Journal of Cellular Physiology, vol. 227, no. 9, pp. 3225-3233, 2012.

[102] V. Turinetto, E. Vitale, and C. Giachino, "Senescence in human mesenchymal stem cells: functional changes and implications in stem cell-based therapy," International Journal of Molecular Sciences, vol. 17, no. 7, p. 1164, 2016.

[103] F. T. Borges, M. B. Convento, and N. Schor, "Bone marrowderived mesenchymal stromal cell: what next?," Stem Cells and Cloning: Advances and Applications, vol. Volume 11, pp. 77-83, 2018.

[104] A. Chatgilialoglu, M. Rossi, F. Alviano et al., "Restored in vivo-like membrane lipidomics positively influence in vitro features of cultured mesenchymal stromal/stem cells derived from human placenta," Stem Cell Research \& Therapy, vol. 8, no. 1, p. 31, 2017.

[105] C. Cavallini, C. Zannini, E. Olivi et al., "Restoring in vivo-like membrane lipidomics promotes exosome trophic behavior from human placental mesenchymal stromal/stem cells," Cell Transplantation, vol. 27, no. 1, pp. 55-69, 2018.

[106] M. R. Showalter, B. Wancewicz, O. Fiehn et al., "Primed mesenchymal stem cells package exosomes with metabolites 
associated with immunomodulation," Biochemical and Biophysical Research Communications, vol. 512, no. 4, pp. 729735, 2019.

[107] H. Tao, Z. Han, Z. C. Han, and Z. Li, "Proangiogenic features of mesenchymal stem cells and their therapeutic applications," Stem Cells International, vol. 2016, 11 pages, 2016.

[108] D. Kehl, M. Generali, A. Mallone et al., "Proteomic analysis of human mesenchymal stromal cell secretomes: a systematic comparison of the angiogenic potential," npj Regenerative Medicine, vol. 4, no. 1, 2019.

[109] M. Gaetani, C. M. Chinnici, A. P. Carreca, C. di Pasquale, G. Amico, and P. G. Conaldi, "Unbiased and quantitative proteomics reveals highly increased angiogenesis induction by the secretome of mesenchymal stromal cells isolated from fetal rather than adult skin," Journal of Tissue Engineering and Regenerative Medicine, vol. 12, no. 2, pp. e949-e961, 2018.

[110] F. A. Dabrowski, A. Burdzinska, A. Kulesza et al., "Comparison of the paracrine activity of mesenchymal stem cells derived from human umbilical cord, amniotic membrane and adipose tissue," The Journal of Obstetrics and Gynaecology Research, vol. 43, no. 11, pp. 1758-1768, 2017.

[111] H. Wegmeyer, A. M. Bröske, M. Leddin et al., "Mesenchymal stromal cell characteristics vary depending on their origin," Stem Cells and Development, vol. 22, no. 19, pp. 2606-2618, 2013.

[112] S. W. Kim, H. Z. Zhang, C. E. Kim, H. S. An, J. M. Kim, and M. H. Kim, "Amniotic mesenchymal stem cells have robust angiogenic properties and are effective in treating hindlimb ischaemia," Cardiovascular Research, vol. 93, no. 3, pp. 525534, 2012.

[113] W. J. Du, Y. Chi, Z. X. Yang et al., "Heterogeneity of proangiogenic features in mesenchymal stem cells derived from bone marrow, adipose tissue, umbilical cord, and placenta," Stem Cell Research \& Therapy, vol. 7, no. 1, p. 163, 2016.

[114] S. Ohnishi, T. Yasuda, S. Kitamura, and N. Nagaya, "Effect of hypoxia on gene expression of bone marrow-derived mesenchymal stem cells and mononuclear cells," Stem Cells, vol. 25, no. 5, pp. 1166-1177, 2007.

[115] C. Conrad, H. Niess, R. Huss et al., "Multipotent mesenchymal stem cells acquire a lymphendothelial phenotype and enhance lymphatic regeneration in vivo," Circulation, vol. 119, no. 2, pp. 281-289, 2009.

[116] M. E. Baldwin, M. M. Halford, S. Roufail et al., "Vascular endothelial growth factor D is dispensable for development of the lymphatic system," Molecular and Cellular Biology, vol. 25, no. 6, pp. 2441-2449, 2005.

[117] C. C. Shen, B. Chen, J. T. Gu et al., "The angiogenic related functions of bone marrow mesenchymal stem cells are promoted by CBDL rat serum via the Akt/Nrf2 pathway," Experimental Cell Research, vol. 344, no. 1, pp. 86-94, 2016.

[118] M. Gnecchi, H. He, L. G. Melo et al., "Early beneficial effects of bone marrow-derived mesenchymal stem cells overexpressing Akt on cardiac metabolism after myocardial infarction," Stem Cells, vol. 27, no. 4, pp. 971-979, 2009.

[119] P. Mignatti, T. Morimoto, and D. B. Rifkin, "Basic fibroblast growth factor released by single, isolated cells stimulates their migration in an autocrine manner," Proceedings of the National Academy of Sciences of the United States of America, vol. 88, no. 24, pp. 11007-11011, 1991.
[120] M. S. Pepper, N. Ferrara, L. Orci, and R. Montesano, "Potent synergism between vascular endothelial growth factor and basic fibroblast growth factor in the induction of angiogenesis in vitro," Biochemical and Biophysical Research Communications, vol. 189, no. 2, pp. 824-831, 1992.

[121] T. J. Lee, S. H. Bhang, H. S. Yang et al., "Enhancement of long-term angiogenic efficacy of adipose stem cells by delivery of FGF2," Microvascular Research, vol. 84, no. 1, pp. 1$8,2012$.

[122] A. Bronckaers, P. Hilkens, W. Martens et al., "Mesenchymal stem/stromal cells as a pharmacological and therapeutic approach to accelerate angiogenesis," Pharmacology \& Therapeutics, vol. 143, no. 2, pp. 181-196, 2014.

[123] A. Mizukami, C. H. Thomé, G. A. Ferreira et al., "Proteomic identification and time-course monitoring of secreted proteins during expansion of human mesenchymal stem/stromal in stirred-tank bioreactor," Frontiers in Bioengineering and Biotechnology, vol. 7, p. 154, 2019.

[124] S. Rolandsson Enes, E. Åhrman, A. Palani et al., "Quantitative proteomic characterization of lung-MSC and bone marrowMSC using DIA-mass spectrometry," Scientific Reports, vol. 7, no. 1, p. 9316, 2017.

[125] G. D. Kusuma, J. Carthew, R. Lim, and J. E. Frith, "Effect of the microenvironment on mesenchymal stem cell paracrine signaling: opportunities to engineer the therapeutic effect," Stem Cells and Development, vol. 26, no. 9, pp. 617-631, 2017.

[126] L. Kordelas, V. Rebmann, A. K. Ludwig et al., "MSC-derived exosomes: a novel tool to treat therapy-refractory graftversus-host disease," Leukemia, vol. 28, no. 4, pp. 970-973, 2014.

[127] F. G. Teixeira and A. J. Salgado, "Mesenchymal stem cells secretome: current trends and future challenges," Neural Regeneration Research, vol. 15, no. 1, pp. 75-77, 2020.

[128] R. Binato, T. de Souza Fernandez, C. Lazzarotto-Silva et al., "Stability of human mesenchymal stem cells during in vitro culture: considerations for cell therapy," Cell Proliferation, vol. 46, no. 1, pp. 10-22, 2013.

[129] Y. Wang, Z. Zhang, Y. Chi et al., “Long-term cultured mesenchymal stem cells frequently develop genomic mutations but do not undergo malignant transformation," Cell Death \& Disease, vol. 4, no. 12, p. e950, 2013.

[130] N. Riggi, L. Cironi, P. Provero et al., "Expression of the FUSCHOP fusion protein in primary mesenchymal progenitor cells gives rise to a model of myxoid liposarcoma," Cancer Research, vol. 66, no. 14, pp. 7016-7023, 2006.

[131] Y. Jung, J. K. Kim, Y. Shiozawa et al., "Recruitment of mesenchymal stem cells into prostate tumours promotes metastasis," Nature Communications, vol. 4, no. 1, 2013.

[132] W. Timp and G. Timp, "Beyond mass spectrometry, the next step in proteomics," Science Advances, vol. 6, no. 2, p. eaax8978, 2020. 